\title{
Association between Global Assessment of Functioning scores and indicators of functioning, severity, and prognosis in first-time schizophrenia
}

This article was published in the following Dove Press journal:

Clinical Epidemiology

2 September 2016

Number of times this article has been viewed

\author{
Ole Köhler' \\ Henriette Thisted Horsdal ${ }^{2}$ \\ Lone Baandrup ${ }^{3,4}$ \\ Ole Mors ${ }^{1,5}$ \\ Christiane Gasse ${ }^{2}$ \\ 'Psychosis Research Unit, Aarhus \\ University Hospital, Risskov, \\ ${ }^{2}$ National Centre for Register-based \\ Research, Department of Economics \\ and Business Economics, Aarhus \\ University, Aarhus, ${ }^{3}$ Center for \\ Neuropsychiatric Schizophrenia \\ Research, Mental Health Center \\ Glostrup, ${ }^{4}$ Mental Health Center \\ Copenhagen, Copenhagen, ${ }^{5} \mathrm{PSYYCH}$, \\ The Lundbeck Foundation Initiative \\ for Integrative Psychiatric Research, \\ Aarhus University, Aarhus, Denmark
}

Correspondence: Ole Köhler Psychosis Research Unit, Aarhus University Hospital, Skovagervej 2, DK-8240 Risskov, Denmark

Tel +452342066 I

Fax +45 7847 I609

Email karkoe@rm.dk
Background: Assessment of psychosocial functioning in people with schizophrenia is important. The Global Assessment of Functioning (GAF-F) scale represents a widely applied, easy, and quick tool, but its validity and reliability have been debated. The aim was to investigate whether GAF-F scores are associated with other indicators of functioning, severity, and hospitalization. Methods: A Danish population-based cohort study of adults ( $\geq 18$ years) with a recorded GAF-F score at first-time schizophrenia diagnosis during 2004-2011 was performed. The internal validity of GAF-F was evaluated by assessing its association with other baseline measures of functioning and illness severity. Risk of schizophrenia hospitalization within 2 years was evaluated using Cox regression stratified by sex and adjusted for age, year of diagnosis, and inpatient/outpatient status at diagnosis.

Results: We identified 2,837 cases of schizophrenia with a GAF-F score at first-time diagnosis (73.0\% inpatients; $62.6 \%$ males). GAF-F was associated with several baseline measures of functioning and illness severity, such as female sex, being in work, and a longer baseline hospitalization. Lower GAF-F scores were associated with higher hospitalization risk among males (reference GAF-F 61-100): GAF-F 51-60: hazard rate ratio (HRR) $=1.24$ (95\% confidence interval $[\mathrm{CI}]=0.89-1.75)$; GAF-F 41-50: HRR $=1.31(95 \% \mathrm{CI}=0.97-1.77)$; GAF-F 31-40: $\mathrm{HRR}=1.36(95 \% \mathrm{CI}=1.01-1.82) ; \mathrm{GAF}-\mathrm{F} 21-30: \mathrm{HRR}=1.50(95 \% \mathrm{CI}=1.09-2.06)$; and GAF-F $1-20:$ HRR $=2.30(95 \% \mathrm{CI}=1.36-3.90)$, fitting a dose-response relationship $(P=0.031)$. This association was not found in females.

Conclusion: GAF-F at first-time schizophrenia diagnosis showed good internal validity against other measures of functionality in a Danish hospital setting. Severe impairment (as measured by the GAF-F score) at first-time schizophrenia diagnosis was associated with a higher risk of 2-year hospitalization among males, which may indicate sex differences in the course of disease and treatment response.

Keywords: Global Assessment of Functioning, GAF, schizophrenia, hospitalization, incident schizophrenia, early phase treatment

\section{Background}

Schizophrenia is a debilitating disorder with many patients suffering relapses, ${ }^{1-3}$ but the clinical course varies substantially and many patients also experience extended periods with stable symptoms. ${ }^{4,5}$ Owing to the complex nature of schizophrenia, consensus exists on the relevance of including both clinical and psychosocial aspects in disease severity assessment and treatment response evaluations. ${ }^{5,6}$ This may be of particular importance within the first 2 years of illness, that is, among incident patients. ${ }^{5,7}$ 
Clinical trials have indicated that better psychosocial functioning is associated with improved treatment response $^{7-10}$ and a lower risk of hospitalizations. ${ }^{11,12}$ However, study populations in these trials were small, and recent reviews have emphasized the need for studies comprising larger populations and also with a focus on newly diagnosed patients to obtain better knowledge on the entire course after diagnosis. ${ }^{3,13,14}$ Furthermore, measures of psychosocial functioning that are reliable, easy to perform, and not timeconsuming are preferable in everyday clinical practice, where lack of time often complicates assessment with detailed rating scales. ${ }^{15}$ Here, the Global Assessment of Functioning (GAF-F) scale, split version, represents an already frequently applied and easily accessible tool for assessing the psychosocial level of functioning, ranging from 1 to 100 , with higher scores indicating better functioning. ${ }^{16}$ The reliability of GAF-F has been questioned, ${ }^{17,18}$ but recent smaller studies have indicated a good validity of the GAF-F scale. ${ }^{16,19-21}$ Finally, no studies have investigated whether a measure of psychosocial functioning at first-time schizophrenia diagnosis is independently associated with clinical outcome during the early clinical course of schizophrenia.

This prompted us to perform a nationwide study on adult patients with incident schizophrenia with a twofold aim - to investigate whether the GAF-F score at first-time schizophrenia diagnosis is associated with: 1) other indicators of functioning and illness severity, that is, the internal validity of GAF-F and 2) clinical outcome, that is, hospitalization due to schizophrenia.

\section{Methods}

\section{The Danish Schizophrenia Registry}

We performed a population-based cohort study by linking Danish nationwide registries, which are well-established sources for research. ${ }^{22-24}$ The Danish Schizophrenia Registry (DSR) was established in the Danish Health Care System in 2003 and constitutes a registry on selected measures of quality of care in relation to patients diagnosed with schizophrenia (International Classification of Diseases, 10th edition [ICD-10]: F20.0-F20.9). ${ }^{25,26}$ The registry is administered by the Danish Clinical Quality Improvement Programme and publicly financed. Merging with the central personal registration system, covering the entire Danish population, ${ }^{22}$ ensures high coverage and good quality of the data.

Since January 1, 2004, data on eight clinical quality measures have been collected, focusing on monitoring and developing the quality of care and treatment of patients with schizophrenia in psychiatric hospitals and outpatient settings. The DSR was validated in a recent study comparing the information from medical records to the variables registered within the DSR and found an overall high agreement of $>80 \%$, however, with substantial variation across individual measures. ${ }^{25}$ In this previous study, GAF-F was not evaluated in detail.

\section{GAF-F}

The GAF scale evaluates both symptom severity and functioning (GAF-F), ${ }^{16}$ ranking a patient from 1 (lowest score) to 100 (highest score) on both scales. Within the DSR, GAF-F is used and clinicians are obligated to record the score at various times. All hospitalized patients should have their GAF-F score registered at discharge or once a year if hospitalization lasts longer than 1 year. All outpatients should have a GAF-F score recorded once yearly and at discharge from the outpatient clinic.

\section{Study population}

Using the Danish Psychiatric Central Research Register (DPCRR), ${ }^{23}$ we identified all adult patients ( $\geq 18$ years) born after January 1, 1955 and for the first-time diagnosed with schizophrenia (ICD-10: F20.0-F20.9) in an inpatient or outpatient setting between January 1, 2004 and December 31, 2011. This register comprises complete records of all diagnoses assigned at mental hospitals in Denmark (inpatient contacts since 1969 and outpatient contacts since 1995). ${ }^{23}$ The validity of the schizophrenia diagnosis is high. ${ }^{24}$ In the current study, the date of the first schizophrenia diagnosis represents the index date. Among patients diagnosed during an admission, the index date was the discharge date. Among patients diagnosed in an outpatient setting, the index date was the date of the visit in the outpatient clinic where the diagnosis was registered. Among these incident patients, we identified all individuals who were also registered with a GAF-F score within the DSR. We identified all recorded GAF-F scores within 21 days of the index date, that is, between 21 days before and 21 days after, and extracted the GAF-F score closest to the index date. This score represented the GAF-F score at baseline, that is, at first-time schizophrenia diagnosis. We subdivided the patients according to the baseline GAF-F score into the following categories: 61-100 (good functioning); 51-60, 41-50, and 31-40 (moderate impairment); and 21-30 and 1-20 (severe impairment). ${ }^{27}$ The use of the data was approved by the Danish Data Protection Agency, the Danish National Board of Health and Statistics Denmark. Analysis was performed on anonymized register data and thus ethical approval and patient consent was not required. 


\section{Assessment of variables to evaluate internal validity}

We identified information on sex and age. ${ }^{22}$ As indicators of functioning, we identified educational level (primary school, secondary school, and higher education) ${ }^{28}$ occupational status (being in work, outside working force, including being on [sickness] leave or under education, unemployment, old-age pension, and early retirement pension), and marital status (single and married/couple) ${ }^{29}$ As indicators for illness severity, we identified from the DSR information on psychoactive substance abuse within the year prior to the incident schizophrenia diagnosis. In addition, we identified information from the DPCRR on the number of previous psychiatric hospital contacts, time since first psychiatric contact, and the following psychiatric diagnoses prior to the first schizophrenia diagnosis: disorders due to use of alcohol or other psychoactive substances, bipolar disorder, depression, and anxiety disorders.

\section{Assessment of clinical outcome}

The outcome of interest was the first hospitalization due to schizophrenia (ICD-10: F20.0-F20.9) during the first 2 years after the first-time schizophrenia diagnosis by July 31, 2013 in the DPCRR. Hospitalization has been evaluated as a useful proxy for relapse in schizophrenia, and a follow-up of 2 years has been used in previous studies. ${ }^{1}$ The hospitalization had to occur at least 14 days after the first-time schizophrenia diagnosis, that is, after the date of the outpatient contact or after discharge from the index psychiatric hospital contact. Thereby, we avoided hospitalizations shortly after or in connection with initiation of an outpatient contact and discharges, which only lasted few days because of administrative reasons.

\section{Statistical analysis}

First, we used logistic regression analysis to investigate differences in baseline characteristics between individuals with and without a measured GAF-F score. Second, regarding internal validity, that is the association of GAF-F score of $\leq 30$ (severe impairment) with baseline characteristics and other variables representing severity or functioning at first-time schizophrenia diagnosis, we also applied logistic regression analyses. We performed mutual adjusted logistic regression analyses and report odds ratios and $95 \%$ confidence intervals (CIs). Third, we followed all individuals from the index date and for 2 years or until July 1, 2013, hospitalization, death, or emigration. We performed complete case Cox regression analyses to evaluate the relationship between GAF-F at incident schizophrenia diagnosis and risk for hospitalization within 2-year follow-up and report hazard rate ratios (HRRs), including 95\% CI. Wald tests were performed to test for linear relationships. All analyses were adjusted for sex, age at first schizophrenia diagnosis, inpatient or outpatient status at diagnosis, and year of diagnosis. All analyses were performed using STATA 13 via remote access to Statistics Denmark.

\section{Subgroup and sensitivity analysis}

We conducted sex-specific subgroup analyses. Secondly, we stratified by age groups. In addition, we investigated if the risk patterns were dependent on whether the first-time schizophrenia diagnosis occurred during admission or an outpatient contact.

We furthermore performed sensitivity analyses on different time windows for the baseline GAF-F score and investigated if the results were dependent on whether we only identified GAF-F scores within 7 or 14 days of diagnosis.

\section{Results}

We identified 2,837 patients with a GAF-F score registered at first-time schizophrenia diagnosis (Figure 1). Logistic regression analyses showed that patients who were registered with a GAF-F score differed in that these patients more often were inpatients at first-time diagnosis, were older, and had more psychoactive substance abuse (Table S1). Importantly, we found no sex differences or differences in the other important proxies for functioning, such as working, marital status, and other prior psychiatric diagnoses. Hence, the patients included in the current study mostly comprised people admitted at first diagnosis.

Table 1 shows characteristics at first schizophrenia diagnosis for the individuals with a GAF-F score.

\section{Internal validity of GAF-F}

Table 2 illustrates that a higher GAF-F score $(>30$, ie moderate/good functioning) at incident schizophrenia diagnosis was significantly associated with female sex and being in work. Among males only, a prior bipolar disorder diagnosis and older age were associated with higher baseline GAF-F scores, whereas a longer baseline hospitalization was associated with lower baseline GAF-F.

\section{Association of GAF-F scores with risk of hospitalization}

During the first 2 years of follow-up, 1,382 (48.8\%) people were hospitalized due to schizophrenia. Lower GAF-F scores at first-time diagnosis were associated with an increased 


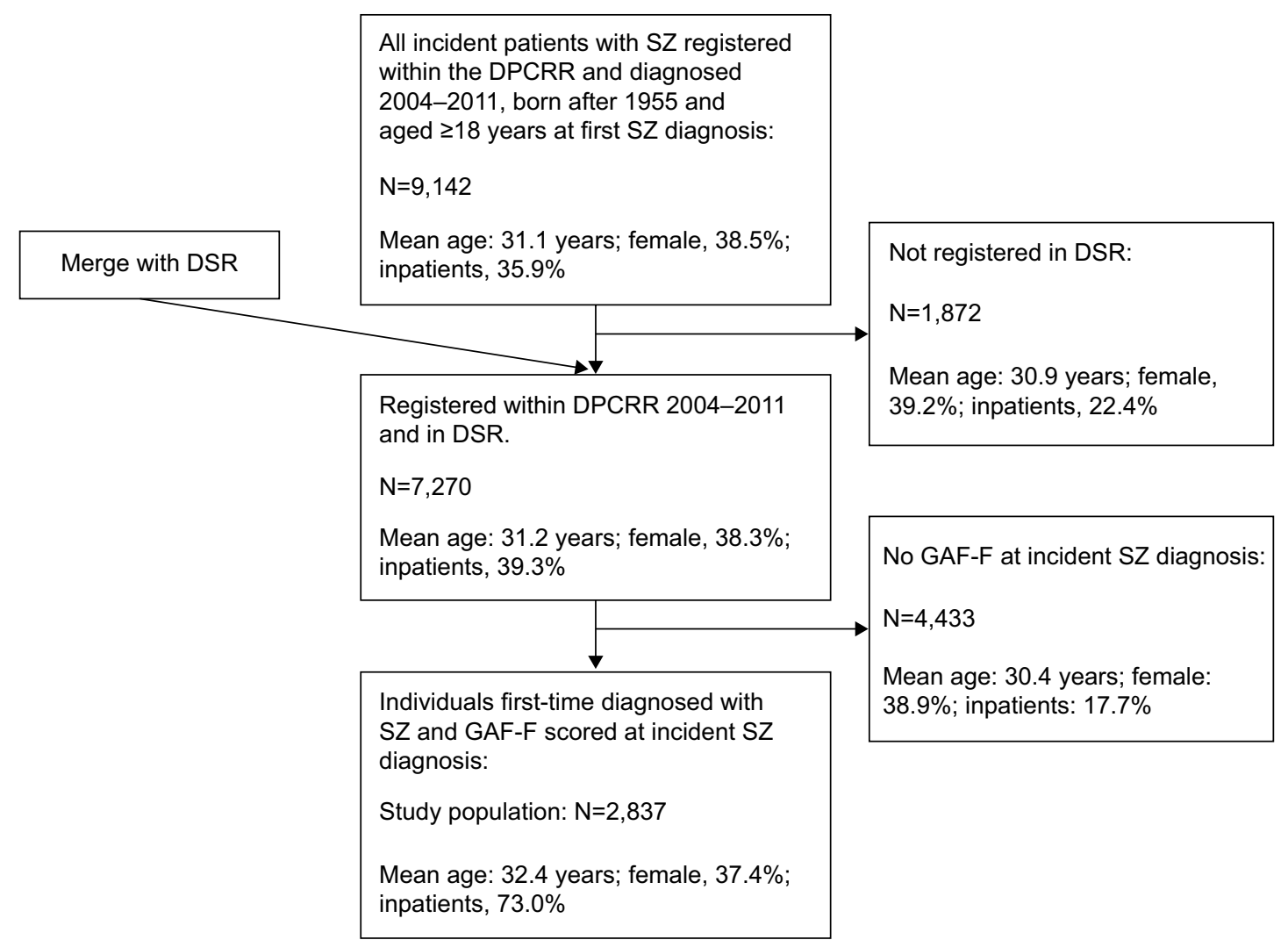

Figure I Flowchart for identification of the study population.

Notes: Patients for the first time diagnosed with SZ between 2004 and 201 I were identified from the DPCRR. Only patients registered in DSR with a GAF-F score between 21 days before or 21 days after the first $S Z$ diagnosis were included in the study.

Abbreviations: DPCRR, Danish Psychiatric Central Research Register; DSR, Danish Schizophrenia Registry; GAF-F, Global Assessment of Functioning; SZ, schizophrenia.

risk of hospitalization (Table 3), indicating a dose-response relationship with lower GAF-F scores predicting higher hospitalization risk (Wald test: $P=0.094$ ). The highest risk was observed among individuals with a GAF-F score between 1 and 20, as compared to GAF-F 61-100: HRR $=1.76(95 \% \mathrm{CI}=1.14-2.72)$. In sex-specific analyses, we found that the risk increase was present only among males (GAF-F 61-100 as reference): GAF-F 51-60: HRR $=1.24$ $(95 \%$ CI $=0.89-1.75)$; GAF-F 41-50: HRR $=1.31(95 \%$ $\mathrm{CI}=0.97-1.77)$; GAF-F 31-40: $\mathrm{HRR}=1.36(95 \% \mathrm{CI}=1.01-$ 1.82); GAF-F 21-30: HRR $=1.50(95 \% \mathrm{CI}=1.09-2.06)$; and GAF-F 1-20: HRR $=2.30(95 \% \mathrm{CI}=1.36-3.90)$, fitting a dose-response relationship $(P=0.031)$ (Table 3$)$. We found no association between GAF-F and hospitalization among females.

\section{Subgroup and sensitivity analyses}

We found similar associations as in the main analyses between lower GAF-F scores and hospitalizations when stratifying on age groups and when comparing individuals diagnosed during admission with individuals diagnosed in an outpatient setting. All sensitivity analyses applying different time windows for identification of the GAF-F score at incident schizophrenia diagnosis supported our primary results associating lower GAF-F scores with an increased hospitalization risk in males. In more detail, when using GAF-F scores registered within 7 days of first-time schizophrenia diagnosis, we found that the risk for hospitalization increased among 1,631 males with decreasing GAF-F scores (GAF-F 61-100 as reference): GAF-F 51-60: HRR $=1.24(95 \% \mathrm{CI}=0.87-1.75)$; GAF-F 41-50: HRR $=1.34$ (95\% CI $=0.98-1.84)$; GAF-F 31-40: HRR $=1.40$ (95\% CI =1.03-1.90); GAF-F 21-30: HRR $=1.50(95 \% \mathrm{CI}=1.07-2.09)$; and GAF-F $1-20: \mathrm{HRR}=2.29$ (95\% CI $=1.32-3.99)$, fitting a dose-response relationship $(P=0.047)$. When using GAF-F scores registered within 14 days of first-time schizophrenia diagnosis, we found that the risk for hospitalization increased among 1,702 males with decreasing GAF-F scores (GAF-F $61-100$ as reference): GAF-F 51-60: HRR $=1.21(95 \% \mathrm{CI}=0.87-1.70)$; GAF-F 41-50: HRR $=1.32(95 \% \mathrm{CI}=0.97-1.80)$; GAF-F 31-40: HRR $=1.34$ (95\% CI =1.00-1.81); GAF-F 21-30: HRR $=1.47(95 \% \mathrm{CI}=1.07-2.04)$; and GAF-F 1-20: HRR $=2.23(95 \% \mathrm{CI}=1.30-3.82)$, fitting a dose-response 
Table I Baseline characteristics of the study population, overall and according to sex

\begin{tabular}{|c|c|c|c|}
\hline Baseline characteristics & Total, N (\%) & Males N (\%) & Females N (\%) \\
\hline Total & $2,837(100)$ & I,777 (62.6) & I,060 (37.4) \\
\hline Mean age, years (SD) & $32.4(9.9)$ & $32.3(9.7)$ & $32.8(10.2)$ \\
\hline \multicolumn{4}{|l|}{ Age group, years } \\
\hline $18-24$ & $887(31.3)$ & $557(31.3)$ & $330(31.1)$ \\
\hline $25-29$ & $474(16.7)$ & $310(17.5)$ & $164(15.5)$ \\
\hline $30-39$ & $708(25.0)$ & $453(25.5)$ & $255(24.1)$ \\
\hline $40+$ & $768(27.1)$ & $457(25.7)$ & $311(29.3)$ \\
\hline \multicolumn{4}{|l|}{ Education } \\
\hline Primary school & $1,650(63.2)$ & $1,069(66.2)$ & $581(58.3)$ \\
\hline Secondary school & $309(11.8)$ & $180(1 \mid .2)$ & $129(12.9)$ \\
\hline Higher education & $652(25.0)$ & $365(22.6)$ & $287(28.8)$ \\
\hline \multicolumn{4}{|l|}{ Work status } \\
\hline Working & $610(22.1)$ & $370(21.5)$ & $240(23.0)$ \\
\hline Outside working force & $\mathrm{I}, 460(52.8)$ & $942(54.7)$ & $518(49.6)$ \\
\hline Early retirement pension & $695(25.1)$ & $409(23.8)$ & $286(27.4)$ \\
\hline \multicolumn{4}{|l|}{ Marital status } \\
\hline Married/couple & $595(21.2)$ & $285(16.1)$ & $310(29.4)$ \\
\hline Single & 2,229 (78.9) & I,483 (83.9) & $746(70.6)$ \\
\hline \multicolumn{4}{|l|}{ Index year } \\
\hline 2004 & $282(9.9)$ & $|8|(10.2)$ & I0I (9.5) \\
\hline 2005 & $339(12.0)$ & $211(11.9)$ & $128(12.1)$ \\
\hline 2006 & $295(10.4)$ & $186(10.5)$ & $109(10.3)$ \\
\hline 2007 & $344(12.1)$ & $203(11.4)$ & $14 \mid(13.3)$ \\
\hline 2008 & $4 I I(14.5)$ & $259(14.6)$ & $152(14.3)$ \\
\hline 2009 & $398(14.0)$ & $257(14.5)$ & I4I (I3.3) \\
\hline 2010 & $414(14.6)$ & $26 \mid(\mid 4.7)$ & $153(14.4)$ \\
\hline 2011 & $354(12.5)$ & $219(12.3)$ & $135(12.7)$ \\
\hline \multicolumn{4}{|l|}{ Length of hospitalization at schizophrenia diagnosis } \\
\hline Outpatient & $766(27.0)$ & $464(26.1)$ & $302(28.5)$ \\
\hline Hospitalized & $2,07 \mid(73.0)$ & $1,313(73.9)$ & $758(71.5)$ \\
\hline $\mathrm{I}-30$ days & $774(37.4)$ & $488(37.2)$ & $286(37.7)$ \\
\hline $31-90$ days & $693(33.5)$ & $416(31.7)$ & $277(36.5)$ \\
\hline $90+$ days & $604(29.1)$ & $409(31.2)$ & $195(25.7)$ \\
\hline Mean years (SD) since first psychiatric contact & $4.98(6.35)$ & $4.71(6.30)$ & $5.42(6.39)$ \\
\hline \multicolumn{4}{|l|}{ Previous psychiatric contacts } \\
\hline 1 & $331(11.7)$ & $225(12.7)$ & $106(10.0)$ \\
\hline 2 & $326(11.5)$ & $212(11.9)$ & $114(10.8)$ \\
\hline $3+$ & $2,180(76.8)$ & $\mathrm{I}, 340(75.4)$ & $840(79.3)$ \\
\hline \multicolumn{4}{|c|}{ Psychoactive substance abuse in the year prior to diagnosis } \\
\hline Yes & I, $189(41.9)$ & $939(52.8)$ & $249(23.5)$ \\
\hline No & I,648 (58.1) & $838(47.2)$ & $811(76.5)$ \\
\hline \multicolumn{4}{|l|}{ Prior psychiatric diagnoses ${ }^{b}$} \\
\hline No diagnosis ${ }^{\mathrm{b}}$ & $1,218(42.9)$ & $734(4 \mid .3)$ & $484(45.7)$ \\
\hline Any diagnosis ${ }^{\mathrm{b}}$ & $1,619(57.1)$ & $\mathrm{I}, 043(58.7)$ & $576(54.3)$ \\
\hline Alcohol abuse & $412(14.5)$ & $314(17.7)$ & $98(9.3)$ \\
\hline Substance abuse & $805(28.4)$ & $660(37.1)$ & $145(13.7)$ \\
\hline Bipolar disorder & $117(4.1)$ & $59(3.3)$ & $58(5.5)$ \\
\hline Depression & $707(24.9)$ & $330(18.6)$ & $377(35.6)$ \\
\hline Anxiety disorder & $330(11.6)$ & $183(10.3)$ & 147 (13.9) \\
\hline
\end{tabular}

Notes: aNumber of contacts to the psychiatric hospital system, including outpatient clinics, prior to the first schizophrenia diagnosis. ${ }^{b} \mathrm{We}$ included the following diagnoses: alcohol abuse, substance abuse, bipolar disorder, depression, and anxiety disorder. Individuals could have had other psychiatric diagnoses.

Abbreviation: SD, standard deviation.

relationship $(P=0.051)$. Within the two above-mentioned sensitivity analyses among females $(\mathrm{N}=980$ and $\mathrm{N}=1,017$ when using GAF-F scores identified within 7 and 14 days, respectively), we found no increased risk of hospitalization depending on lower GAF-F scores.

\section{Discussion}

The present study evaluated GAF-F scores at first-time schizophrenia diagnosis among 2,837 incident patients with schizophrenia. We found that GAF-F scores at the first-time schizophrenia diagnosis were associated with other measures 
Table 2 Correlation between a low GAF-F score (GAF-F $\leq 30$ versus GAF-F $>30$ ), and other variables reflecting psychosocial functioning and illness severity, at first schizophrenia diagnosis, calculated using logistic regression

\begin{tabular}{|c|c|c|c|c|c|c|}
\hline \multirow[b]{2}{*}{ Covariate (\% completeness of data) } & \multicolumn{2}{|c|}{ Overall } & \multicolumn{2}{|c|}{ Males } & \multicolumn{2}{|c|}{ Females } \\
\hline & OR & $95 \% \mathrm{Cl}$ & OR & $95 \% \mathrm{Cl}$ & OR & $95 \% \mathrm{Cl}$ \\
\hline Age (100\%) & 0.99 & $0.97-1.00$ & 0.98 & $0.97-0.99$ & 1.00 & $0.97-1.03$ \\
\hline Female sex $(100 \%)$ & 0.76 & $0.58-0.98$ & - & - & - & - \\
\hline \multicolumn{7}{|l|}{ Education (92.1\%) } \\
\hline Primary school & 1.0 & Ref & 1.0 & Ref & 1.0 & Ref \\
\hline Secondary school & 0.77 & $0.53-1.12$ & 0.65 & $0.40-1.05$ & 1.00 & $0.55-1.82$ \\
\hline Higher education & 0.82 & $0.6 I-I . I I$ & 0.91 & $0.62-1.33$ & 0.72 & $0.44-1.21$ \\
\hline \multicolumn{7}{|l|}{ Work status (97.5\%) } \\
\hline Early retirement pension & 1.0 & Ref & 1.0 & Ref & 1.0 & Ref \\
\hline Outside working force & 0.43 & $0.3 I-0.60$ & 0.42 & $0.28-0.62$ & 0.45 & $0.26-0.78$ \\
\hline In work & 0.26 & $0.17-0.39$ & 0.24 & $0.14-0.41$ & 0.28 & $0.14-0.58$ \\
\hline Being married/couple (99.5\%) & 0.83 & $0.61-1.13$ & 0.80 & $0.53-1.21$ & 0.84 & $0.52-1.35$ \\
\hline \multicolumn{7}{|l|}{ Length of baseline hospitalization ( $100 \%)$} \\
\hline Outpatient & 1.0 & Ref & 1.0 & Ref & 1.0 & Ref \\
\hline $\mathrm{I}-30$ days & 1.12 & $0.8 \mathrm{I}-1.56$ & 1.14 & $0.75-1.73$ & 1.09 & $0.64-1.85$ \\
\hline $31-90$ days & 1.04 & $0.74-1.47$ & 1.17 & $0.76-1.80$ & 0.83 & $0.47-1.48$ \\
\hline $90+$ days & 1.91 & $1.39-2.63$ & 2.42 & $1.64-3.58$ & 1.09 & $0.60-1.99$ \\
\hline Time since first psychiatric contact ( $100 \%)$ & 1.00 & $0.98-1.02$ & 1.01 & $0.98-1.03$ & 0.99 & $0.95-1.03$ \\
\hline Psychoactive substance abuse within the year & 0.92 & $0.69-1.22$ & 0.94 & $0.67-1.32$ & 0.93 & $0.55-1.58$ \\
\hline \multicolumn{7}{|l|}{ prior to diagnosis (96.0\%) } \\
\hline \multicolumn{7}{|l|}{ Prior psychiatric diagnoses } \\
\hline Bipolar disorder (100\%) & 0.49 & $0.23-1.04$ & 0.30 & $0.09-0.98$ & 0.72 & $0.27-1.90$ \\
\hline Major depression (100\%) & 1.01 & $0.76-1.33$ & 1.10 & $0.76-1.57$ & 0.97 & $0.62-1.52$ \\
\hline Anxiety (100\%) & 0.73 & $0.49-1.06$ & 0.75 & $0.47-1.20$ & 0.64 & $0.33-1.22$ \\
\hline Alcohol/substance abuse (100\%) & 1.31 & $0.97-1.77$ & 1.24 & $0.88-1.75$ & 1.46 & $0.78-2.72$ \\
\hline
\end{tabular}

Note: Bold numbers represent statistically significant results.

Abbreviations: $\mathrm{Cl}$, confidence interval; GAF-F, Global Assessment of Functioning, functioning; OR, odds ratio; Ref, reference.

of functionality and disease severity. Furthermore, lower GAF-F scores were associated with an increased risk of hospitalizations in males within 2 years of follow-up following a dose-response relationship. Males with a GAF-F score of 1-20, compared to males with a GAF-F score of 61-100, had a more than twofold increased risk of hospitalization. All analyses were adjusted for important covariates and supported by several sensitivity analyses.

\section{Validity of GAF-F}

Previous studies have questioned the reliability of GAF-F, ${ }^{17,18}$ whereas more recent studies have emphasized its usefulness and good validity. ${ }^{20,21}$ However, these studies were rather small. We included 2,837 incident patients with schizophrenia and showed that the GAF-F score at the first-time schizophrenia diagnosis was associated with other important measures of psychosocial functioning, such as poorer occupational ability and a longer baseline hospitalization. Hence, our results support previous studies suggesting a good validity of the GAF-F scale ${ }^{20,21}$ and the usefulness of measuring the level of psychosocial functioning among incident patients with schizophrenia. ${ }^{16,19}$ Importantly, the GAF-F score has been shown to correlate well with other frequently applied measures of psychosocial functioning and symptom severity, such as the Social and Occupational Functioning Assessment Scale, the Clinical Global Impression Scale, the Positive and Negative Syndrome Scale, and the Brief Psychiatric Rating Scale. ${ }^{20,21}$ Thus, our results support previous studies ${ }^{20,21}$ suggesting that the GAF-F scale represents a valid, inexpensive, and not time-consuming tool for assessing the functional level of the patient. Importantly, a high inter-rater reliability can be achieved with little training of the raters, ${ }^{16}$ which is an important factor in everyday clinical work often suffering of time to perform more time-consuming rating scales..$^{15}$

\section{GAF-F and the early clinical course of illness}

Previous studies have identified several predictors for hospitalization with schizophrenia, such as male sex, comorbidities, lack of early antipsychotic response, and younger age at diagnosis. ${ }^{1-3}$ Furthermore, studies have indicated the importance of including both clinical and psychosocial aspects in disease severity assessment and treatment response evaluations early after the diagnosis, for example, within the first 2 years of illness. ${ }^{5,7}$ In addition, studies have discussed the importance of focusing on modifiable risk factors 
Table 3 HRR for hospitalization depending on baseline GAF-F score at incident SZ diagnosis, subdivided depending on sex

\begin{tabular}{|c|c|c|c|c|}
\hline $\begin{array}{l}\text { All } \\
\text { GAF-F at }\end{array}$ & N (\%) & Person-time (years) & Relapse (\% of total) & $\operatorname{HRR}^{\mathrm{a}}(95 \% \mathrm{Cl})^{\mathrm{d}}$ \\
\hline Total & $2,837(100)$ & $3,697.5$ & I,382 (48.8) & \\
\hline $61-100$ & $222(7.9)$ & 305.8 & $93(41.7)$ & 1.00 (ref) \\
\hline $5 I-60$ & $394(13.9)$ & 520.1 & $183(46.5)$ & $1.17(0.92-1.52)$ \\
\hline $4 I-50$ & $749(26.4)$ & 985.7 & $375(50.1)$ & $1.26(1.01-1.58)$ \\
\hline $31-40$ & $1,065(37.6)$ & $1,360.2$ & $532(50.0)$ & $1.30(1.04-1.61)$ \\
\hline $21-30$ & $363(12.8)$ & 477.1 & I 74 (47.9) & $1.28(1.00-1.65)$ \\
\hline $\mathrm{I}-20$ & $44(1.6)$ & 48.6 & $26(59.1)$ & $1.76(1.14-2.72)$ \\
\hline $\begin{array}{l}\text { Males } \\
\text { GAF-F at }\end{array}$ & $\mathbf{N}(\%)$ & Person-time (years) & Relapse (\% of total) & $\operatorname{HRR}^{\mathrm{b}}(95 \% \mathrm{Cl})^{\mathrm{d}}$ \\
\hline Total & $\mathrm{I}, 777(100)$ & $2,390.4$ & $826(46.5)$ & \\
\hline $61-100$ & $|4|(7.9)$ & 201.5 & $53(37.6)$ & 1.00 (ref) \\
\hline $5 I-60$ & $227(12.8)$ & 312.5 & $99(43.6)$ & $1.24(0.89-1.75)$ \\
\hline $4 I-50$ & $432(24.3)$ & 589.2 & $203(47.0)$ & 1.31 (0.97-I.77) \\
\hline $31-40$ & $687(38.7)$ & 919.8 & $321(46.9)$ & $1.36(1.01-1.82)$ \\
\hline $21-30$ & $26 I(14.7)$ & 336.9 & $|3|(50.2)$ & $1.50(1.09-2.06)$ \\
\hline $\mathrm{I}-20$ & $29(1.6)$ & 30.5 & $19(65.5)$ & $2.30(1.36-3.90)$ \\
\hline $\begin{array}{l}\text { Females } \\
\text { GAF-F at }\end{array}$ & $\mathbf{N}(\%)$ & Person-time (years) & Relapse (\% of total) & HRR $^{c}(95 \% \mathrm{Cl})^{d}$ \\
\hline Total & $1,060(100)$ & $\mathrm{I}, 308.4$ & $556(52.5)$ & \\
\hline $61-100$ & $82(7.7)$ & 105.3 & $4 \mid(50.0)$ & 1.00 (ref) \\
\hline $5 I-60$ & $167(15.8)$ & 208.1 & $83(49.7)$ & $1.06(0.73-1.54)$ \\
\hline $4 I-50$ & $313(29.5)$ & 390.4 & $170(54.3)$ & $1.19(0.84-1.67)$ \\
\hline $31-40$ & 381 (35.9) & 448.2 & $212(55.6)$ & $1.23(0.88-1.72)$ \\
\hline $21-30$ & $102(9.6)$ & 138.3 & $43(42.2)$ & $0.89(0.58-1.36)$ \\
\hline $\mathrm{I}-20$ & $15(1.4)$ & 18.1 & $7(46.7)$ & $1.06(0.73-1.54)$ \\
\hline
\end{tabular}

Notes: $\mathrm{a}=0.094$ for linear relationship. ${ }^{\mathrm{b} P}=0.03 \mathrm{I}$ for linear relationship. ${ }^{\mathrm{C}} \mathrm{P}=0.37$ for linear relationship. ${ }^{\mathrm{d}}$ The results are adjusted for age, sex, year of diagnosis, and inpatient or outpatient status at diagnosis. Data in bold indicate significant results.

Abbreviations: $\mathrm{Cl}$, confidence interval; GAF-F, Global Assessment of Functioning; HRR, hazard rate ratio; SZ, schizophrenia; ref, reference.

(eg, the functional level of the patient) instead of nonmodifiable risk factors for worse treatment response (eg, sex and age). ${ }^{3}$ The importance of assessment of the functional level already during the early clinical course has been demonstrated in several studies, ${ }^{3,5,7}$ indicating that among first-episode psychosis patients, females reach higher psychosocial functional levels than males during 5 years of follow-up. ${ }^{30}$ However, evidence is sparse regarding single measures of psychosocial functioning in association with the risk for hospitalization among incident patients with schizophrenia. Only a few clinical trials have studied the association between psychosocial functioning and outcome measures, mostly among prevalent patients with schizophrenia and without being sex-specific. ${ }^{11,12,31}$ Patients with schizophrenia who were rehospitalized, compared to patients who were not, had lower GAF scores ${ }^{12}$ and lower social functioning as measured by the quality of life scale. ${ }^{11}$ Worse premorbid social functioning among patients with first-episode psychosis, as measured by the premorbid adjustment scale, has been found to be associated with poorer response to 6-week antipsychotic treatment ${ }^{9}$ and with worse clinical course after $5^{7,8}$ and 10 years. ${ }^{10}$ Nevertheless, these studies were limited by small patient populations, and the present study represents the largest investigation of the association between one measure of the psychosocial level of functioning and the early clinical course, including 2,837 patients with a first-time schizophrenia diagnosis. Thus, our results extend the knowledge on the importance of low functioning in schizophrenia. We found that lower GAF-F scores among males with a first-time schizophrenia diagnosis were associated with a higher 2-year risk of schizophrenia hospitalization fitting a dose-response relationship. The reason for this association only in males may be manifold. One possible explanation might be a sex difference in liability of hospitalization as an indicator for relapse, that is, that care givers have a lower threshold for admitting males than females and/or that females in general have a higher level of social functioning and thus might not require rehospitalization as often as males. ${ }^{32}$

\section{Strengths and limitations}

The strengths are the population-based design and the thoroughly validated Danish registers. ${ }^{22-24}$ The DSR has 
an overall high validity, ${ }^{25}$ and we found sufficient internal validity for GAF-F in the current study. Our findings are furthermore strengthened as we identified incident patients with schizophrenia from the Danish Psychiatric Central Research Register $^{23}$ and stratified into different GAF-F levels at the time of the first-time schizophrenia diagnosis. Inclusion of incident patients yields the advantage of generalization of the results, as information on the entire course after diagnosis is available, as opposed to prevalent cohorts.

Regarding limitations, GAF-F scores were more frequently registered among inpatients. Thus, the current study population consisted mostly of inpatients, that is, the most severely ill patients, indicating selection bias. Second, other measures of psychosocial functioning than the GAF-F are used in different clinical psychiatric settings, but recent studies have found that some of the most frequently applied measures used in psychiatry correlated well with GAF-F. ${ }^{20,21}$ Third, the GAF-F score does not necessarily reflect the functionality at onset of hospitalization, but rather a score of the patient's status during the days and/or weeks around discharge. It can be assumed that GAF-F scores recorded at the beginning of hospitalization would have been lower. However, this may have rather underestimated than overestimated our association. Still, the above mentioned limitations may possibly have resulted in misclassification of functionality as indicated by the GAF-F score. Nevertheless, our results were significant in sensitivity analyses with different time windows of the assessments of the baseline GAF-F score.

\section{Conclusion}

Among 2,837 incident patients with schizophrenia, GAF-F at first-time schizophrenia diagnosis was associated with other measures of functioning in a Danish hospital setting. Furthermore, lower GAF-F scores were associated with an increased risk of 2-year hospitalization among males in a dose-response relationship, which may indicate sex differences in the course of disease and treatment response. Thereby, our results expand the findings associating psychosocial measures of functioning with the early clinical course of schizophrenia.

\section{Acknowledgment}

The research leading to these results received funding from the European Community's Seventh Framework Programme (FP7/2007-2013) under grant agreement no 279227. The authors would like to thank the CRESTAR consortium (development of pharmacogenomic biomarkers for schizophrenia).

\section{Author contributions}

All authors contributed toward data analysis, drafting and revising the paper and agree to be accountable for all aspects of the work.

\section{Disclosure}

The authors report no conflicts of interest in this work.

\section{References}

1. Olivares JM, Sermon J, Hemels M, Schreiner A. Definitions and drivers of relapse in patients with schizophrenia: A systematic literature review. Ann Gen Psychiatry. 2013;12(1):32.

2. Suzuki T, Uchida H, Takeuchi H, Tsuboi T, Hirano J, Mimura M. A review on schizophrenia and relapse - a quest for user - friendly psychopharmacotherapy. Hum Psychopharmacol. 2014;29(5):414-426.

3. Carbon M, Correll CU. Clinical predictors of therapeutic response to antipsychotics in schizophrenia. Dialogues Clin Neurosci. 2014;16(4):505-524.

4. Andreasen NC, Carpenter WT Jr, Kane JM, Lasser RA, Marder SR, Weinberger DR. Remission in schizophrenia: Proposed criteria and rationale for consensus. Am J Psychiatry. 2005;162(3):441-449.

5. Jaaskelainen E, Juola P, Hirvonen N, et al. A systematic review and meta-analysis of recovery in schizophrenia. Schizophr Bull. 2013;39(6):1296-1306.

6. Leucht $\mathrm{S}$, Lasser R. The concepts of remission and recovery in schizophrenia. Pharmacopsychiatry. 2006;39(5):161-170.

7. Albert N, Bertelsen M, Thorup A, et al. Predictors of recovery from psychosis analyses of clinical and social factors associated with recovery among patients with first-episode psychosis after 5 years. Schizophr Res. 2011;125(2-3):257-266.

8. Bertelsen M, Jeppesen P, Petersen L, et al. Course of illness in a sample of 265 patients with first-episode psychosis - five-year follow-up of the Danish OPUS trial. Schizophr Res. 2009;107(2-3):173-178.

9. Crespo-Facorro B, de la Foz VO, Ayesa-Arriola R, et al. Prediction of acute clinical response following a first episode of non affective psychosis: Results of a cohort of 375 patients from the Spanish PAFIP study. Prog Neuropsychopharmacol Biol Psychiatry. 2013;44:162-167.

10. Austin SF, Mors O, Secher RG, et al. Predictors of recovery in first episode psychosis: The OPUS cohort at 10 year follow-up. Schizophr Res. 2013;150(1):163-168.

11. Olfson M, Ascher-Svanum H, Faries DE, Marcus SC. Predicting psychiatric hospital admission among adults with schizophrenia. Psychiatr Serv. 2011;62(10):1138-1145.

12. Schennach R, Obermeier M, Meyer S, et al. Predictors of relapse in the year after hospital discharge among patients with schizophrenia. Psychiatr Serv. 2012;63(1):87-90.

13. Marshall M, Rathbone J. Early intervention for psychosis. Cochrane Database Syst Rev. 2011;15(6):CD004718.

14. Nordentoft M, Rasmussen JO, Melau M, Hjorthoj CR, Thorup AA. How successful are first episode programs? A review of the evidence for specialized assertive early intervention. Curr Opin Psychiatry. 2014;27(3):167-172.

15. Brissos S, Molodynski A, Dias VV, Figueira ML. The importance of measuring psychosocial functioning in schizophrenia. Ann Gen Psychiatry. 2011;10:18.

16. Jones SH, Thornicroft G, Coffey M, Dunn G. A brief mental health outcome scale-reliability and validity of the global assessment of functioning (GAF). Br J Psychiatry. 1995;166(5):654-659.

17. Aas IH. Guidelines for rating global assessment of functioning (GAF). Ann Gen Psychiatry. 2011;10:2.

18. Aas IH. Global assessment of functioning (GAF): Properties and frontier of current knowledge. Ann Gen Psychiatry. 2010;9:20.

19. Startup M, Jackson MC, Bendix S. The concurrent validity of the global assessment of functioning (GAF). Br J Clin Psychol. 2002;41(Pt 4): $417-422$. 
20. Samara MT, Engel RR, Millier A, Kandenwein J, Toumi M, Leucht S. Equipercentile linking of scales measuring functioning and symptoms: Examining the GAF, SOFAS, CGI-S, and PANSS. Eur Neuropsychopharmacol. 2014;24(11):1767-1772.

21. Suzuki T, Uchida H, Sakurai H, et al. Relationships between global assessment of functioning and other rating scales in clinical trials for schizophrenia. Psychiatry Res. 2015;227(2-3):265-269.

22. Pedersen CB. The Danish civil registration system. Scand J Public Health. 2011;39(7 Suppl):22-25.

23. Mors O, Perto GP, Mortensen PB. The Danish psychiatric central research register. Scand J Public Health. 2011;39(7 Suppl): 54-57.

24. Uggerby P, Ostergaard SD, Roge R, Correll CU, Nielsen J. The validity of the schizophrenia diagnosis in the Danish psychiatric central research register is good. Dan Med J. 2013;60(2):A4578.

25. Pedersen CG, Gradus JL, Johnsen SP, Mainz J. Challenges in validating quality of care data in a schizophrenia registry: Experience from the Danish national indicator project. Clin Epidemiol. 2012;4 201-207.
26. Mainz J, Krog BR, Bjornshave B, Bartels P. Nationwide continuous quality improvement using clinical indicators: The Danish national indicator project. Int J Qual Health Care. 2004;16 (Suppl 1):i45-i50.

27. American Psychiatric Association. Diagnostic and Statistical Manual of Mental Disorder, 4th ed. Washington DC: American Psychiatric Association; 1994

28. Jensen VM, Rasmussen AW. Danish education registers. Scand J Public Health. 2011;39(7 Suppl):91-94.

29. Petersson F, Baadsgaard M, Thygesen LC. Danish registers on personal labour market affiliation. Scand J Public Health. 2011;39(7 Suppl):95-98.

30. Thorup A, Albert N, Bertelsen M, et al. Gender differences in first-episode psychosis at 5-year follow-up - two different courses of disease? results from the OPUS study at 5-year follow-up. Eur Psychiatry. 2014;29(1):44-51.

31. Agid O, Siu CO, Pappadopulos E, Vanderburg D, Remington G. Early prediction of clinical and functional outcome in schizophrenia. Eur Neuropsychopharmacol. 2013;23(8):842-851.

32. Angermeyer MC, Goldstein JM, Kuehn L. Gender differences in schizophrenia: Rehospitalization and community survival. Psychol Med. 1989;19(2):365-382. 


\section{Supplementary material}

Table SI Association of characteristics of individuals who were GAF-F scored at first-time schizophrenia diagnosis ( $N=2,827)$ and individuals who did not receive a GAF-F score $(\mathrm{N}=4,433)$, using logistic regression

\begin{tabular}{|c|c|c|c|c|}
\hline Covariate (\% completeness of data) & $\begin{array}{l}\text { With GAF-F score } \\
N=2,837\end{array}$ & $\begin{array}{l}\text { Without GAF-F score } \\
N=4,433\end{array}$ & OR & $95 \% \mathrm{Cl}$ \\
\hline Mean age, years (SD) (I00\%) & $32.4(9.9)$ & $30.4(9.4)$ & 1.02 & I.01; I.03 \\
\hline Female sex $(100 \%)$ & $\mathrm{I}, 060(37.4)$ & I,723 (38.9) & 1.09 & $0.96 ; 1.24$ \\
\hline \multicolumn{5}{|l|}{ Work status (97.9\%) } \\
\hline Early retirement pension & $695(25.1)$ & $755(17.4)$ & 1.0 & Ref \\
\hline Outside working force & $\mathrm{I}, 460(52.8)$ & $2,574(59.2)$ & 0.89 & $0.76 ; 1.06$ \\
\hline In work & $610(22.1)$ & $\mathrm{I}, 02 \mathrm{I}(23.5)$ & 1.03 & $0.85 ; 1.24$ \\
\hline Being married/couple (99.1\%) & $595(21.2)$ & I,I $157(26.4)$ & 0.95 & $0.82 ; 1.09$ \\
\hline \multicolumn{5}{|l|}{ Length of baseline hospitalization ( $100 \%)$} \\
\hline Outpatient & $766(27.0)$ & $3,647(82.3)$ & 1.0 & Ref \\
\hline $\mathrm{I}-30$ days & $774(37.4)$ & $382(8.6)$ & 12.27 & $7.18 ; 20.98$ \\
\hline $31-90$ days & $693(33.5)$ & $202(4.6)$ & 19.18 & $10.08 ; 36.49$ \\
\hline $90+$ days & $604(29.1)$ & $202(4.6)$ & 30.38 & 15.66; 58.94 \\
\hline $\begin{array}{l}\text { Psychoactive substance abuse within the } \\
\text { year prior to diagnosis }(92.4 \%)\end{array}$ & $1,189(41.9)$ & $1,419(32.0)$ & 2.09 & $1.36 ; 3.21$ \\
\hline \multicolumn{5}{|l|}{ Prior psychiatric diagnoses (100\%) } \\
\hline Bipolar disorder & $117(4.1)$ & $129(2.9)$ & 1.07 & $0.78 ; 1.46$ \\
\hline Major depression & 707 (24.9) & $1,090(24.6)$ & 1.04 & $0.91 ; 1.20$ \\
\hline Anxiety & $330(11.6)$ & $549(12.4)$ & 0.93 & $0.77 ; 1.12$ \\
\hline Alcohol/substance abuse & $975(34.4)$ & I, $199(27.1)$ & 1.14 & $0.97 ; 1.33$ \\
\hline
\end{tabular}

Notes: Data are presented as $n$ (\%). Bold numbers represent statistically significant results. OR adjusted for all factors shown, including interaction terms for hospitalization and age, and hospitalization and psychoactive substance abuse.

Abbreviations: $\mathrm{Cl}$, confidence interval; GAF-F, Global Assessment of Functioning; OR, odds ratio; SD, standard deviation; Ref, reference.

\section{Publish your work in this journal}

Clinical Epidemiology is an international, peer-reviewed, open access, online journal focusing on disease and drug epidemiology, identification of risk factors and screening procedures to develop optimal preventative initiatives and programs. Specific topics include: diagnosis, prognosis, treatment, screening, prevention, risk factor modification,

Submit your manuscript here: https://www.dovepress.com/clinical-epidemiology-journa systematic reviews, risk and safety of medical interventions, epidemiology and biostatistical methods, and evaluation of guidelines, translational medicine, health policies and economic evaluations. The manuscript management system is completely online and includes a very quick and fair peer-review system, which is all easy to use. 